\title{
Regulatory Landscape in the Arab Region: Introduction
}

\author{
Henry Silverman
}

\section{Current Regulatory Situation in the Arab Region}

Research governance systems vary between the different countries in the Arab Region and embrace some variant of the models discussed in Chapter 15. In those countries who operate a "loose" regulatory climate, concerns will exist regarding the adequacy of the protection of the rights and welfare of research participants. Such concerns will suggest the need for the development of robust national regulatory systems that include adequate oversight mechanisms and robust ethics review of research. The aim of Part IV is to describe the regulatory systems that exist in several countries in the Arab region.

The case studies in this section serve several purposes. First, to show the range of the regulatory frameworks governing research in several of the Arabic countries. This review will show that several countries lack robust systems, but several have reached a level of sophistication that conform with international standards and are comparable to the systems in many western countries. Second, those countries who have achieved a robust regulatory system can thus provide a "model" to which other Arabic countries with developing research governance systems can emulate.

H. Silverman ( $\square)$

University of Maryland, Baltimore, MD, USA

e-mail: hsilverm@medicine.umaryland.edu 